\title{
Revise and conquer
}

In between the twin certainties of reject and accept lies a large range of decisions covered by the blanket term of Revise. Here we discuss how to deal with this uncertain result.

R eceiving an e-mail informing you that your paper has been accepted for publication is a clear-cut - and hopefully pleasant - decision. Rejection, on the other hand, tends to be considerably less pleasant, but it is at least still definitive. But what about the more uncertain instances where the paper is not accepted, but a revised version is requested?

This covers a wide range of cases, from papers where only a small amount of extra data is needed to push it over the line, to cases where it is far from clear as to whether the work will ultimately be published. Frequent examples of the latter include studies where the strength of evidence for the claims made in the paper is in doubt this is always the most important issue for us, and the one on which your editor and reviewers will be most focussed.

In making the decision, the editor will go through all the points raised by the reviewers, in addition to their own assessment. Criticisms listed by the referees are mentally sorted into one of two camps: editorial concerns which relate to whether or not the work should be published in this particular journal, and technical concerns which relate to whether the work is sound and the conclusions are supported - and hence, whether the work as it stands is publishable in any journal.

Upon receiving a request for revision, the first thing, of course, is to methodically read through all the referee reports. Once you have done this, it is worth reflecting on whether you think you can reasonably address these comments. It may well be that it is not possible or practical to do so, and submitting the work elsewhere would be the smartest choice. In that case, it is important to inform the editorial office of your decision, so that your file can be closed, avoiding the risk of infringing dual submission policies. Submitting a revised version that does not address the key points is likely to end up costing time for no additional benefit. And remember that the reviewer reports can be a great resource to strengthen your claims and improve the message of the paper.

What about discussing the decision with the editor? Unless the reports are very self-explanatory, it is likely that the editor will already have mentioned some of the key points in the decision letter you received. Often, they will have specifically highlighted the points that they consider to be the most critical, or what requests they consider optional or beyond the scope of the current work. But you may want to raise additional points, and do not hesitate to do so. It may be that some experiments raised by the referees will not be possible, or have already been tried. Or perhaps you feel that some of the work requested will require a great deal of effort, but not lead to meaningful results. Feel free to talk to your editor about this. That is not so say that you can argue out of important, though laborious, work, nor that the editor will necessarily agree with you. A revised version that contains no new data, but merely argues against all of the reviewer comments, is unlikely to be successful. But, it can be useful to open the discussion in mutual good faith.

\section{"It may be that some experiments raised by the referees will not be possible, or have already been tried. Or perhaps you feel that some of the work requested will require a great deal of effort, but not lead to meaningful results. Feel free to talk to your editor about this."}

Hopefully this will have equipped you with a clear plan of what is needed to move the process forward. If you feel that this is a route you are happy to take, then it is likely that more experiments or analysis will be needed. As mentioned above, it is likely to be more beneficial to carry out this work than to argue against it, unless you have very strong arguments in your favour. We typically do not set strict time limits on how long the revisions should take, and we are happy to extend deadlines for revisions if you discuss this with your editor. Another thing worth noting is that even with extended revisions, we believe that the original submission date should be maintained: in the overwhelming majority of cases the submitted date of the published article will be that of the first submission to the journal. Only in exceptional cases - such as where the paper has changed far beyond what is typical during even an intensive peerreview process, and the links to the original work are somewhat tenuous do we consider resetting the submission date to that of the revised version.

Then, sooner or later, you will have a revised version ready to submit. At this point it is worth putting some effort into your response to reviewers. Your feelings towards the reviewers may depend on how constructive you found their earlier feedback. Do, however, bear in mind that these are your colleagues, and that it is highly unlikely that their criticism - even when harsh - was intended personally. So, try to take the comments objectively, and respond in a similar manner. This is not just a matter of professional courtesy: an irritated tone can immediately put a referee on the defensive, and make the entire process more confrontational than it needs to be. Sometimes we contact authors and ask - or recommend - that they moderate the tone in order to maintain an objective atmosphere. (People sometimes ask why we request that authors moderate their tone, but not reviewers. This is incorrect; we can, and do, ask referees to edit reports where they become overly personal or discourteous). If you feel strongly about some points made by a reviewer, a better strategy is to raise any objections directly with the editor, rather than having the argument play out through the medium of a rebuttal letter and reviewer report.

And then, once you have done all you can, it is time to resubmit. From our side, we will go through your work in detail, mostly likely discuss it with the reviewers, and get back to you as soon as we can. Hopefully, this time, with a more definitive answer.

Published online: 13 June 2019 https://doi.org/10.1038/s41929-019-0316-5 